\title{
Isolation and evolutionary analyses of porcine epidemic diarrhea virus in Asia
}

\author{
Wan Liang ${ }^{1,2}$, Danna Zhou ${ }^{1}$, Chao Geng ${ }^{1,3}$, Keli Yang ${ }^{1}$, Zhengying Duan ${ }^{1}$, Rui Guo ${ }^{1}$, Wei Liu ${ }^{1}$, Fangyan Yuan ${ }^{1}$, \\ Zewen Liu ${ }^{1}$, Ting Gao ${ }^{1}$, Ling Zhao ${ }^{2}$, Dongwan Yoo ${ }^{4}$, Yongxiang Tian ${ }^{\text {Corresp. } 1}$ \\ ${ }^{1}$ Key Laboratory of Prevention and Control Agents for Animal Bacteriosis (Ministry of Agriculture), Institute of Animal Husbandry and Veterinary Science, \\ Hubei Academy of Agricultural Sciences, Wuhan, China \\ 2 Key Laboratory of Preventive Veterinary Medicine of Hubei Province, College of Veterinary Medicine, Huazhong Agricultural University, Wuhan, China \\ 3 College of Animal Sciences, Yangtze University, Jinzhou, China \\ 4 Department of Pathobiology, College of Veterinary Medicine, University of Illinois at Urbana-Champaign, Champaign, United States \\ Corresponding Author: Yongxiang Tian \\ Email address: tyxanbit@163.com
}

Porcine epidemic diarrhea virus (PEDV) is a leading cause of diarrhea in pigs worldwide. Virus isolation and genetic evolutionary analysis allow investigations into the prevalence of epidemic strains and provide data for the clinical diagnosis and vaccine development. In this study, we investigated the genetic characteristics of PEDV circulation in Asia through virus isolation and comparative genomics analysis. A PEDV strain designated HB2018 was isolated from a pig in a farm experiencing a diarrhea outbreak. The complete genome sequence of HB2018 was 28,138 bp in length. Phylogenetic analysis of HB2018 and 207 PEDVs in Asia showed that most PEDV strains circulating in Asia after 2010 belong to genotype GII, particularly GII-a. The PEDV vaccine strain CV777 belonged to GI, and thus, unmatched genotypes between CV777 and GII-a variants might partially explain incomplete protection by the CV777-derived vaccine against PEDV variants in China. In addition, we found the $\mathrm{S}$ protein of variant strains contained numerous mutations compared to the S protein of CV777, and these mutations occurred in the N-terminal domain of the $\mathrm{S}$ protein. These mutations may influence the antigenicity, pathogenicity, and neutralization properties of the variant strains. 


\section{Isolation and evolutionary analyses of porcine epidemic diarrhea virus in Asia}

2 Wan Liang ${ }^{1,2}$, Danna Zhou ${ }^{1}$, Chao Geng ${ }^{1,3}$, Keli Yang ${ }^{1}$, Zhengying Duan ${ }^{1}$, Rui Guo ${ }^{1}$, Wei Liu

3 , Fangyan Yuan ${ }^{1}$, Zewen Liu ${ }^{1}$, Ting Gao ${ }^{1}$, Ling Zhao ${ }^{2}$, Dongwan Yoo ${ }^{4}$ and Yongxiang Tian $41, *$

$6 \quad{ }^{1}$ Key Laboratory of Prevention and Control Agents for Animal Bacteriosis (Ministry of 7 Agriculture), Institute of Animal Husbandry and Veterinary, Hubei Academy of Agricultural 8 Sciences, Wuhan, China; liangwan521521@163.com (WL); zdn_66@126.com (DZ);

9 a18163131821@163.com (CG); keliy6@126.com (KY); zy001d@sina.com (ZD);

10 hlguorui@163.com (RG); liuwei85@126.com（WLiu); hbxms@126.com (FY);

11 liuzwen2004@sina.com (ZL); gt20170719@163.com (TG); tyxanbit@163.com (YT)

$12{ }^{2}$ Key Laboratory of Preventive Veterinary Medicine of Hubei Province, College of Veterinary

13 Medicine, Huazhong Agricultural University, Wuhan, China; liangwan521521@163.com (WL);

14 lingzhao@mail.hzau.edu.cn (LZ).

$15 \quad{ }^{3}$ College of Animal Sciences, Yangtze University, Jinzhou, China; a18163131821@163.com $16 \quad(\mathrm{CG})$

$17{ }^{4}$ Department of Pathobiology, College of Veterinary Medicine, University of Illinois at Urbana18 Champaign, Champaign, United States; dyoo@illinois.edu (DY)

19

$20 *$ Corresponding author. E-mail address: tyxanbit@,163.com (Y. Tian) 


\section{ABSTRACT}

22 Porcine epidemic diarrhea virus (PEDV) is a leading cause of diarrhea in pigs worldwide. Virus

23 isolation and genetic evolutionary analysis allow investigations into the prevalence of epidemic

24 strains and provide data for the clinical diagnosis and vaccine development. In this study, we

25 investigated the genetic characteristics of PEDV circulation in Asia through virus isolation and

26 comparative genomics analysis. A PEDV strain designated HB2018 was isolated from a pig in a

27 farm experiencing a diarrhea outbreak. The complete genome sequence of HB2018 was 28,138

28 bp in length. Phylogenetic analysis of HB2018 and 207 PEDVs in Asia showed that most PEDV

29 strains circulating in Asia after 2010 belong to genotype GII, particularly GII-a. The PEDV

30 vaccine strain CV777 belonged to GI, and thus, unmatched genotypes between CV777 and GII-

31 a variants might partially explain incomplete protection by the CV777-derived vaccine against

32 PEDV variants in China. In addition, we found the $\mathrm{S}$ protein of variant strains contained

33 numerous mutations compared to the S protein of CV777, and these mutations occurred in the N-

34 terminal domain of the S protein. These mutations may influence the antigenicity, pathogenicity, 35 and neutralization properties of the variant strains.

36 Keywords: Porcine epidemic diarrhea virus; virus isolation; genetic evolutionary analysis; amino 37 acid mutations; Asia 


\section{INTRODUCTION}

Porcine epidemic diarrhea (PED) is a high contagious and devastating disease resulting in the watery diarrhea in suckling pigs with high mortality and morbidity (Zhang et al. 2019). The causative agent of PED, the porcine epidemic diarrhea virus (PEDV), is an enveloped, singlestranded, positive-sense RNA virus belonging to the genus Alphacoronavirus in the family Coronaviridae (Woo et al. 2012). PEDV possesses a 28-kb genome which encodes seven proteins including ORF1a, ORF1b, spike (S) glycoprotein, ORF3 hypothetical protein, envelop (E) protein, membrane (M) protein and nucleocapsid protein (Guo et al. 2019). Among these proteins, the S protein plays a key role in interaction between the virus and host cells. S protein consists of 1383amino acids (Aziz et al.), and amino acid changes in S protein may lead to antigenic variations and affect the virus virulence (Gong et al. 2018; Suzuki et al. 2018). Therefore, this protein is commonly used as an important target for analyzing genetic variations and molecular epidemiology of PEDV (Hsueh et al. 2020).

PED outbreaks have been reported continuously in China since 1973. PED was well controlled since administration of a CV777-derived vaccine (Chen et al. 2019a; Wang et al. 2016a). However, recent outbreaks of PED in China since 2010 was due to the re-emergence of PEDV, and the continuous spread of the virus during the last 10 years has resulted in serious economic losses in the pig industry in Asian countries (Yang et al. 2013). In these outbreaks, inactivated vaccines and attenuated live vaccines, which were derived from CV777, were used to control the disease but neither of them provided effective protection (Sun et al. 2012; Zhou et al. 2012). Moreover, the virus has evolved since 2010 (Guo et al. 2019; Hsu et al. 2018; Sun et al. 2019), and acquisition of whole genome features of PEDV provides a convenient tool for the tracking of PEDV epidemiology (Chen et al. 2019b). In addition, virus isolation and genetic analysis allow 
61 investigations on the prevalence of epidemic strains and will provide information for diagnosis

62 and vaccine developments ( $\mathrm{Li}$ et al. 2018). In this study, we isolated a highly pathogenic PEDV

63 strain HB2018 from a pig in a farm experiencing PED outbreaks in Hubei province, China, and

64 determined its complete genome sequence. By comparing the HB2018 genome sequence with the

65 sequences of 207 PEDV isolates circulating in Asia, which were publicly available in the Genbank

66 data base, this study also aims to elucidate the evolutionary and genetic characteristics of PEDV

67 currently circulation in different regions of Asia.

\section{MATERIALS AND METHODS}

\section{Virus detection and isolation}

In 2018, an outbreak of diarrhea occurred in a CV777-vaccinated pig farm (numbers of sows $\geq 100$ ) in Hubei Province in China. Many pigs in the farm suffered from severe watery diarrhea, and some of them died. Samples of intestinal tissues were collected from dead pigs and sent to the Veterinary Diagnostic Laboratory of Hubei Academy of Agricultural Sciences in Wuhan, China, for diagnosis. Tissues were immersed with Dulbecco's modified Eagle medium (DMEM; Gibco, Grand Island, NY, USA), and were then homogenized using a QIAGEN TissueLyser II (QIAGEN, Dusseldorf, Nordrhein-Westfalen, Germany). The sample homogenates were then frozen at -80 ${ }^{\circ} \mathrm{C}$ and thawed for three times. After that, the supernatants were filtered through a $0.22-\mu \mathrm{m}$ membrane and were harvested for RNA and virus isolation. Total RNAs were extracted using TRIzol (Thermo, Waltham, MA, USA) and were reverse transcribed to cDNA using a Thermo Scientific First Strand cDNA Synthesis kit (Thermo, Waltham, MA, USA). Viral nucleic acids were detected by RT-PCR assays using the cDNA as templates and the primers specific for PEDV (F: 5'-TTCGGTTCTATTCCCGTTGATG-3', R: 5'-CCCATGAAGCACTTTCTCACTATC-3'), 
83 TGEV (transmissible gastroenteritis virus) (F: 5'-TTACAAACTCGCTATCGCATGG-3', R: 5'-

84 TCTTGTCACATCACCTTTACCTGC-3') and PoRV (porcine rotavirus) (F: 5'-

85 CCCCGGTATtGAATATACCACAGT-3'， R: 5'-TTTCTGTTGGCCACCCTTTAGT-3'),

86 respectively.

87 Vero cells (Purchased from ATCC, Manassas, VA, USA) were used for virus isolation. In

88 brief, homogenate supernatants and trypsin $(5 \mu \mathrm{g} / \mathrm{ml})$ were inoculated into monolayers of Vero

89 cells, which were then incubated in a $37{ }^{\circ} \mathrm{C}$ incubator supplemented with $5 \% \mathrm{CO}_{2}$. Cells with

90 obvious cytopathic effects (CPEs) were harvested, thawed, and refrozen multiple times. The

91 harvested virus suspension was then inoculated into newly prepared Vero cells for passages, and

92 the propagation was continuously performed for 20 passages (F20). Virus RNA was extracted

93 every five passage for RT-PCR detection of the virus nucleic acids.

94 Virus titration and serum neutralization

95 Virus titers were measured on 96-well plates using 10-fold serial dilutions of culture

96 supernatant in triplicate per dilution to determine the quantity of viruses required to produce CPEs

97 in $50 \%$ of cells. After incubating for enough time, no more CPEs appeared, and TCID50 was

98 calculated using the Reed-Muench method (Reed \& Muench 1983). The virus titer was also

99 determined by plaque assay using Vero cells and expressed as plaque-forming units (PFU) per mL.

100 The serum neutralization (SN) test was performed in 96-well plates with inactivated serum

101 collected from the guinea pigs infected with the vaccine strain CV777. The virus was diluted in

102 serum-free DMEM to make 200 TCID in a $50 \mu \mathrm{L}$ volume, and mixed with $50 \mu \mathrm{L}$ of 2 -fold serial

103 dilution serum. The mixture was added to cells cultured in 96 -well plates and incubated at $37^{\circ} \mathrm{C}$

104 for $1 \mathrm{~h}$. After removing the mixture and thoroughly washing three times with PBS, the cells were 
105 incubated at $37^{\circ} \mathrm{C}$ with $5 \% \mathrm{CO}_{2}$ for 2 days. Neutralization titers were calculated as the reciprocal

106 of the highest dilution of serum that inhibits CPEs.

\section{Genome sequencing and annotation}

108 Genomic RNA was extracted using the TAKARA RNA extraction kit (Takara, Kusatsu,

109 Shiga, Japan) following the manufacture instruction. The quantity and quality of the extracted

110 RNA were measured by using a Nanodrop spectrophotometer (Thermo, Waltham, MA, USA). The

111 RNA was then subjected to reverse transcription for cDNA using a cDNA synthesis kit (Thermo,

112 Waltham, MA, USA). Genome sequencing was performed with a paired-end library constructed

113 by using a NEB-Next ${ }^{\circledR}$ DNA Library Prep Master Mix Set for Illumina (NEB, Ipswich, MA, USA)

114 and subsequently sequenced on an Illumina NextSeq 500 with $2 \times 150$ paired end sequencing

115 chemistry. After filtering, the clean reads were assembled using SPAdes v3.10.1 (Bankevich et al.

116 2012) and assembled sequences were mapped to the reference genome. The prediction of the genes

117 and proteins were conducted with Prokka v1.12 and RAST Serve (http://rast.nmpdr.org) (Aziz et

118 al. 2008). The complete genome sequence as well as its annotations were deposited into NCBI

119 GenBank under the accession number MT166307.

120 Comparative genomics and bioinformatical analysis

121 The NCBI data was search for "porcine epidemic diarrhea virus" and a total of 207 complete

122 genome sequences were publicly available for PEDV isolates representing different parts of Asia

123 (See Table S1 in supplementary materials). All of these 207 sequences were downloaded for

124 further analysis. The average nucleotide sequence identity between the genomes of HB2018 and

125 CV777 was calculated by ANI calculator (Goris et al. 2007). Sequence alignments were performed

126 using MAFFT v7.4.02 (Katoh \& Standley 2013). Nucleotide sequence similarity and the putative 
127 recombination sites was assessed by SimPlot v.3.5.1 (Lole et al. 1999), with a sliding window size

128 of $500 \mathrm{bp}$, step size of 100 nucleotides, and 1,000 bootstrap replicates, using gap-stripped

129 alignments and the F84 (ML) distance model. Phylogenetic trees based on complete genome

130 sequences were generated by using MEGA X software with 1,000 bootstrapping (Kumar et al.

131 2018).The evolutionary history was inferred by using the Maximum Likelihood method and

132 Tamura-Nei model (Tamura \& Nei 1993). Initial tree(s) for the heuristic search were obtained

133 automatically by applying Neighbor-Join and BioNJ algorithms to a matrix of pairwise distances

134 estimated using the Maximum Composite Likelihood (MCL) approach, and then selecting the

135 topology with superior log likelihood value. The tree is drawn to scale, with branch lengths

136 measured in the number of substitutions per site. A maximum likelihood tree was also generated

137 using the BEAST 2 package (version 2.6.3) (Bouckaert et al. 2019). Gamma correction for site

138 heterogeneity and the GTR model (Gatto et al. 2007) were selected for the tree generation. Both

139 of the trees were annotated and visualized by using the iTOL v.4 online tool (Interactive Tree of

140 Life, http://itol.embl.de/) (Letunic \& Bork 2019). Single nucleotide polymorphisms (SNPs)

141 between two genome sequences were determined by the MAUVE package (version 2.4.0) (Darling

142 et al. 2004), and the coding effect of these SNPs were analyzed using a previously reported local

143 Perl command (Peng et al. 2016). Protein structure was generated using SWISS-MODEL

144 (Waterhouse et al. 2018). Protein N-glycosylation sites were predicted using online software

145 (http://www.cbs.dtu.dk/services/NetNGlyc/). Threshold values of greater than 0.5 and Jury

146 agreement 9/9 were used for the high-specificity N-glycosylation sites determination (Sagesser et

147 al. 1997).

148 RESULTS

149 Isolation of PEDV HB2018 and its genomic characteristics 
RT-PCR detection of the viral nucleic acids revealed that the intestinal samples from pigs

151 suffered and died from severe watery diarrhea were positive for PEDV but negative for TGEV and

152 PoRV (Figure S1 in supplementary materials). Through virus isolation and purification using Vero

153 cells and determination of PEDV nucleic acids using RT-PCR, a PEDV strain was finally

154 recovered and designated HB2018. The TCID50/0.1 mL value of HB2018 was $10^{5.3}$. The complete

155 genome sequence of PEDV strain HB2018 was 28,138 bp in length. This 2.8-kb genome contained

156 seven open reading frames (ORFs): ORF1a (nucleotide positions 281 to 12634), ORF1b (positions

157 12,664 to 20,625), S gene (positions 24,782 to 25,456), ORF3 (positions 25,675 to 25,667), E gene

158 (positions 25,437 to 25,667), $\mathrm{M}$ gene (positions 25,675 to 26,355), and $\mathrm{N}$ gene (positions 26,367

159 to 27,692$)$.

160 Phylogenetic analysis based on the complete genome sequence showed that HB2018 was

161 phylogenetically distinct from the vaccine strain CV777 (Figure 1A). According to the genotyping

162 system based on a full-length genomic sequence analysis (Guo et al. 2019; Wang et al. 2016a),

163 HB2018 and CV777 belonged to two different genotype: HB2018 was assigned as a type GII strain

164 while CV777 was a GI strain (Figure 1A). The average nucleotide identity between the genomes

165 of HB2018 and CV777 (GenBank accession no. AF353511) was 96.06\% (Figure S2 in

166 supplementary materials). The ORF1, ORF3, E, M, and N genes of HB2018 as well as their

167 encoding proteins were highly homologous to those of CV777 (nucleotide identity $\geq 95 \%$ for

168 genes; amino acid similarity $\geq 95 \%$ for proteins) (Figure 1B; Table 1). However, the identity of

169 the $\mathrm{S}$ genes and proteins between the two strains was relatively low: the homology for nucleotide

170 and amino acid sequences between HB2018 and CV777 were 93.76\% and 93.44\%, respectively

171 (Figures 1B \& 1C; Table 1). SNP analysis determined a total of 946 SNPs in the genome sequence

172 of HB2018 when compared to the genome sequence of the reference strain CV777. Among these 
173 SNPs, 925 SNPs including 262 non-synonymous substitutions and 663 synonymous substitutions

174 were located with the ORF regions, with an overall ratio of nonsynonymous to synonymous

175 substitutions (dN/dS) of 0.39 (Table 2). The dN/dS ratios in each of the ORFs encoded by the

176 HB2018 genome ranged from 0.15 to 0.61 , with the S protein had the highest $\mathrm{dN} / \mathrm{dS}$ ratio (Table 177 2).

178 Compared to the S protein of CV777, the S protein of HB2018 had changes, deletions, and/or 179 insertions of amino acids at multiple sites (Table S2 and Figure S3 in supplementary materials).

180 Notably, most of these mutations occurred in the N-terminal domain (NTD, 19-233aa) of the S

181 protein (Figure 1C; Figure S3 in supplementary materials). Interestingly, some of these mutations

182 were located within the neutralizing epitopes of PEDV [COE (499-638), SS2 (748-755), SS6

183 (764-771) and 2C10 (1368-1374)]. In addition, these mutations led to a structural change at some 184 parts of the HB2018 S protein compared to the CV777 S protein (Figures 1D\&1E).

\section{Phylogenetic analysis of Aisan PEDV isolates}

186 To explore the phylogenic relationships of the PEDVs currently circulating in Asia, we

187 generated two maximum likelihood trees based on the whole genome sequences, either by using

188 the MEGA X software with the Tamura-Nei model (Figure 2A) or by using the BEAST 2 package

189 with the GTR model (Figure 2B). Both of the results revealed that the 208 PEDV strains in Asia,

190 representing the 207 genome sequences publicly available in NCBI and the HB2018 sequence was

191 divided into two genogroups: GI (classical) and GII (variant). Interestingly, isolates in China

192 before 2010 and the vaccine strain CV777 were included within the GI genogroup. However, most

193 of the PEDV isolates from China as well as the other Asian countries after 2010 belonged to GII

194 genogroup (Figures 2A\&2B). The phylogenetic trees also showed that the two genogroups

195 consisted of several subgroups: the genogroup GI was divided into two subgroups, GI-a and GI-b, 
196 while the genogroups GII was divided into three subgroups, GII-a, GII-b, and GII-c (Figures

197 2A\&2B). The GI-a and GI-b subgroups included isolates from China before 2010 and several

198 Chinese isolates between 2010 and 2015 (Figures 2A\&2B). Most of the GII isolates from China

199 and South Korea and all GII isolates from Japan were included within the GII-a subgroup, while

200 less proportion of the Chinese GII isolates and most of the GII-a isolates from Southeast Asia

201 (Vietnam and Thailand) were included within GII-b subgroup (Figures 2A\&2B). Interestingly, the

202 GII-c subgroup only consisted of isolates from China (Figures 2A\&2B).

203 By analyzing isolation years and genogroups of PEDVs, the history of PEDV and the 204 evolution in China are speculated. Between 1986 and 2008, only five PEDV strains were 205 sequenced in China, and all of them belonged to G1 (Figures 2A\&2B). However, the number of 206 PEDV sequences increased significantly after 2010 (Figure 2C). While several PEDV sequences 207 belonged to genogroup GI after 2010, most sequences from China were GII strains (Figures 208 2A\&2B\&2C).

\section{Analysis on the S protein}

210 Compared to the S proteins of the Chinese GI-a strains, amino acid changes, deletions, and/or

211 insertions were observed at multiple sites within the S proteins of the Chinese GI-b strains (Table

212 S3 and Txt S1 in supplementary materials). Compared to the S proteins of the Chinese GI strains,

213 the S proteins of the Chinese GII strains commonly had amino acid changes, deletions, and/or 214 insertions at several sites (Table S4 and Txt S1 in supplementary materials). Most these mutations 215 occurred in S-NTD (19-233aa) of the S protein (Figure 3A; Txt S1 in supplementary materials).

216 Compared to S proteins of the Chinese GII-a strains, S proteins of most isolates from Japan,

217 South Korea, and Vietnam did not contain characteristic amino acid mutations, with the exception 218 of S proteins of two Japanese strains (NIG-2/JPN/2014 and KMM-1/JPN/2014) which had a 
219 continuous deletion of 194 amino acids at sites 23-216 (Figure 3B; Txt S2 in supplementary

220 materials). In addition, four isolates from Japan (GenBank accession num. LC063844 LC063847)

221 and three isolates from South Korea (KNU-141112-S DEL5, KNU-141112-S DEL5ORF3, KNU-

222 1406-1) had a continuous deletion of 5 amino acids (GENQG) at sites 56-60 in their S proteins

223 compared to the S proteins of the Chinese GII-a strains (Figure 3B; Txt S2 in supplementary

224 materials). In addition to the GII-a strains, several isolates from China, South Korea, Thailand, and

225 Vietnam were GII-b strains (Figure 1). Compared to S proteins of most of the Chinese GII-b

226 strains, S proteins of the GII-b isolates from Thailand and Vietnam had amino acid changes at sites

227 130-131 (SI $\rightarrow \mathrm{DN}), 182(\mathrm{Y} \rightarrow \mathrm{H}), 287(\mathrm{I} \rightarrow \mathrm{M}), 324(\mathrm{~N} \rightarrow \mathrm{D}), 327(\mathrm{~S} \rightarrow \mathrm{A}), 358(\mathrm{~A} \rightarrow \mathrm{T}), 367(\mathrm{I} \rightarrow \mathrm{T})$,

$228433(\mathrm{D} \rightarrow \mathrm{G})$, 558-559 $(\mathrm{TN} \rightarrow \mathrm{PT}), 1287(\mathrm{E} \rightarrow \mathrm{K})$, and $1317(\mathrm{~L} \rightarrow \mathrm{F})$ (Txt S3 in supplementary 229 materials).

230 The N-glycosylation sites in the S proteins of the Asian strains studied were investigated 231 herein. Bioinformatical analysis revealed that most of the 208 Asian isolates contained 7 9 high232 specificity $\mathrm{N}$-glycosylation sites in their S proteins. When combining all high-specificity N233 glycosylation sites determined and deleting the duplicates, eleven sites appeared in most isolates, 234 including 57NSTW60, 112NATA115, 127NKTL130, 212NVTS215, 320NDTS323, 235 347NSSD350, 510NITV513, 552NVTN555, 777NISI780, 1245NKTL1248, and 1257NRTG1260 236 (Table 3). Among these sites, 212NVTS215, 777NISI780, and 1245NKTL1248 were conserved 237 in almost all of the 208 Asian strains. However, the N-glycosylation sites at sites 57-60 of some 238 strains were "NSSS" rather than "NSTW". This is because the S proteins of these strains had the 239 59QGVN62 deletion compared to the S proteins of the other strains. Due to the amino acids 240 changes, the N-glycosylation sites at sites $347-350$ in some strains also changed from "NSSD" to 241 "NSSN" or "NSTN". The 510NITV513 and 552NVTN555 N-glycosylation sites were missing in 
242 S proteins of the isolates from South Korea and Vietnam. Due to the large deletion of amino acids

243 at sites 23-216, the 57NSTW60, 112NATA115, 127NKTL130, and 212NVTS215 N-

244 glycosylation sites were missing in S proteins of two Japanese strains NIG-2/JPN/2014 and KMM-

$2451 / \mathrm{JPN} / 2014$, and seven N-glycosylation sites, including 130NDTS133, 157NSSN160, 246 494NVTS497, 549NCTE552, 587NISI590, 1055NKTL1058, and 1067NRTG1070 were retained 247 in these two strains.

\section{Analysis of the ORF3-E-M-N proteins}

249 Unlike the S protein, ORF3 is a conserved protein among the PEDV isolates (Wang et al. 250 2016b). However, several PEDVs were found to have characteristic amino acid mutations in the

251 ORF3 (Figure 4). Compared to ORF3 proteins of many G1 strains and all GII strains, nine GI-a 252 PEDVs (ZJUG12013, 85-7, 85-7-mutant 1, 85-7-mutant 2, 85-7-mutant 3, 85-7-mutant 4, 85-7253 mutant 5, 85-7-A40, and 85-7-C40) had a continuous deletion of 70 amino acids at their N-terminal 254 (positions 1-70) of ORF3; two GI-a strains (85-7-mutant 2 and 85-7-mutant 4) had a continuous 255 deletion of 47 amino acids at their C-terminal (positions 178-224) of ORF3; while nine GI-b strains 256 (JS2008, AH-M, SD-M, SQ2014, SC1402, HLJBY, PEDV-SX, JSLS-12015 and JS-22015) had a 257 continuous deletion of 133 amino acids at their C-terminal (positions 92-224) of ORF3 (Figure 4). 258 Compared to ORF3 proteins of many GI and GII strains, ORF3 protein of a GII-a strain 259 (CHSXYL2016) had a continuous deletion of 14 amino acids at positions 211-224; ORF3 protein 260 of another GII-a strain (NW17) had a continuous deletion of 6 amino acids (DLYLAI) at positions 261 168-173; while ORF3 proteins of six GII-b strains (YN15, YN30, YN60, YN90, YN144, YN200) 262 had a continuous deletion of 79 amino acids at their C-terminal (positions 146-224) (Figure 4). 263 Compared to ORF3 proteins of the GII-a isolates, more than half of the GII-b isolates had amino 264 acid changes at positions $25(\mathrm{~L} \rightarrow \mathrm{S}), 70(\mathrm{I} \rightarrow \mathrm{V}), 80(\mathrm{~V} \rightarrow \mathrm{F}), 107(\mathrm{C} \rightarrow \mathrm{F}), 168(\mathrm{D} \rightarrow \mathrm{N})$, and 182 
$265(\mathrm{Q} \rightarrow \mathrm{H})($ Figure 4). Interestingly, these amino acid changes were also found in the ORF3 proteins

266 of many GII-c isolates after 2016.

267 Sequence comparisons revealed that there were no common INDELs or mutations in E 268 proteins of one subgroup of GII strains compared to E proteins of other subgroups of GII strains 269 (Figure 5A). M proteins of most GII-a strains had a glutamine (Q) at site 13; however, all GII-b 270 strains isolated between 2011 and 2012 had a glutamic acid (E) at the same position in their M 271 proteins, and this amino acid change $(\mathrm{Q} \rightarrow \mathrm{E})$ occurred frequently in M proteins of GII-b since 2013

272 (Figure 5B). A similar phenomenon was also observed in the M proteins of the GII-c strains, as 273 most of the GII-c strains isolated before 2016 had a glutamine (Q) at position 13 in their M 274 proteins, but a $\mathrm{Q} \rightarrow \mathrm{E}$ change at position 13 was seen in the $\mathrm{M}$ proteins of more frequently in strains 275 isolated after 2016. In addition, amino acid changes at positions $192(\mathrm{G} \rightarrow \mathrm{S})$ and $214(\mathrm{~S} \rightarrow \mathrm{A})$ 276 appeared simultaneously in M proteins of some GII-b and GII-c strains. Similarly, in N proteins, 277 amino acid changes at positions $216(\mathrm{M} \rightarrow \mathrm{V})$ and $241(\mathrm{R} \rightarrow \mathrm{K})$ appeared simultaneously in many 278 GII strains (Figure 5C).

279 Discussion

280 As an infectious virus attracted great intention, the PEDV strains were frequently reported and 281 isolated in Asia. The virus isolation and genetic analysis will provide important information for 282 PEDV research and vaccine developments. In this study, we isolated a GII-a strain HB2018 and 283 determined its genomic characteristics (Figure 1A). Comparative genomic analysis revealed that 284 the ORF1, ORF3, E, M, and N genes of HB2018 as well as their encoding proteins were highly

285 homologous to those of CV777 (Figure 1B; Table 1). However, a number of SNPs were 
286 determined within these ORFs, with the S proteins showed the highest dN/dS ratio (Table 2). Since

$287 \mathrm{dN} / \mathrm{dS}$ ratio is commonly used as a measure of purifying versus diversifying selection (Rocha et

288 al. 2006), the highest $\mathrm{dN} / \mathrm{dS}$ ratio suggests $\mathrm{S}$ protein is under diversifying selection, and this

289 diversifying selection might be associated with its frequent interaction with host cells.

290 Sequence alignments determined many mutations in the genome sequence of HB2018

291 compared to that of the reference strain CV777. These mutations, especially in the S protein, might

292 be the pathogenic determinants for it, because some deletions and insertions in the S protein may

293 change the antigenicity, pathogenicity and neutralization properties (Chen et al. 2019a; Sagesser

294 et al. 1997; Zhang et al. 2015). The presence of these mutations in the NTD of S protein in HB2018

295 might have an effect on the viral pathogenicity since the S-NTD domain is proposed to be the

296 region relevant to the virulence of PEDV (Hou et al. 2017; Su et al. 2018; Su et al. 2019; Suzuki

297 et al. 2018). In addition, the structural changes led by these mutations in S protein of HB2018

298 might influence the immunogenicity. The distinct phylogenetic relationship between our isolation

299 HB2018 and CV777 might partly explain why vaccination of pigs with CV777 did not provide

300 effective protection against the infection of HB2018 in the vaccinated pig farm (Figures 2A\&2B).

301 The analysis based on isolation years and genogroups of PEDVs in Asia might also revealed the

302 vaccine CV777 did not match with the pandemic PEDV isolations. Before 2010, all the strains in

303 China belonged to GI (Figures 2A\&2B). During this period, PEDV was well controlled in China

304 due to the use of CV777 which was the GI-based vaccine (Chen et al. 2019a; Yang et al. 2013).

305 The phylogenetic analysis of Asian PEDV isolates showed that most of the PEDV isolates from 
306 Asia after 2010 belonged to GII genogroup, while the vaccine CV777 were included within GI

307 genogroup. These findings agree with the results of the other studies (Guo et al. 2019; Wang et al.

308 2016a). The unmatched genotypes between CV777 and PEDV epidemic strains in Asia after 2010

309 could explain why vaccination with CV777 could not stop the outbreak of PED in many Asian

310 countries after 2010 and provide effective protection against the current epidemic strains (Chen et

311 al. 2019a; Puranaveja et al. 2009; Sun et al. 2012; Zhou et al. 2012).

312 With the most reported numbers of PEDV strains, China has more genogroups than other

313 countries. The GII-c subgroup only consisted of isolates from China, these findings are also in

314 agreement with previous studies (Guo et al. 2019; Wang et al. 2016a), suggesting that the

315 genotypes of PEDV strains circulating in China might be more heterogeneous than those of the

316 isolates in other Asian countries. These findings may also explain why PEDV vaccines developed

317 in China contain more than one strains that generally include CV777 and at least one more local

318 GII isolate (http://vdts.ivdc.org.cn:8081/cx/\#). The new emerged PEDV in 2010 might accelerate

319 numerous isolations and sequencing of PEDVs (Li et al. 2012; Yang et al. 2013). In this article, it

320 was found that the number of PEDV sequences increased significantly after 2010 and most

321 sequences were GII strains. These results are in good agreement with the findings of the PEDV

322 epidemiological investigations in China (Chen et al. 2019a; Sun et al. 2018). It has been reported

323 that PEDV GII isolates were more virulent than GI isolates (Vlasova et al. 2014). This might in

324 part explain why the traditional vaccines had no to little effect on the control and spread of PEDV

325 in China after 2010. It is noteworthy that PEDV GII strains are also responsible for the recent 
326 outbreaks of PED in North America and Europe (Choudhury et al. 2016). These findings suggest

327 the circulation of PEDV GII strains also pose a problem to the global pig industry.

328 S protein is the most variable protein of PEDV, the amino acid changes in this protein may

329 lead to virus variation and affect the virus virulence (Gong et al. 2018; Suzuki et al. 2018). The

330 mutations between were found between GI-a strains and GI-b strains, it is still uncertain whether

331 these mutations between them has a biological significance. While, the mutations occurred in S-

332 NTD of the S protein between GI strains and GII strains might in part explain why do the PEDV

333 GII isolates be more pathogenic than the GI isolates (Vlasova et al. 2014), as S-NTD is proposed

334 to be the region relevant to the virulence of PEDV (Hou et al. 2017; Su et al. 2018; Su et al. 2019;

335 Suzuki et al. 2018). It is worthy note that PEDVs with insertions of amino acids at 167-168 and

336 deletions of amino acids at 55-58 and 144 in their S proteins are called S-INDEL strains (Wang et

337 al. 2014). A previous study has found infection of the S-INDEL strains could induce pro-

338 inflammatory cytokines through the non-canonical NF- $\kappa \mathrm{B}$ signaling pathway by activating RIG-

339 I; however, infection of the non-S-INDEL strains suppresses the induction of pro-inflammatory

340 cytokines and type-I interferon production by down-regulation of TLRs and downstream signaling

341 molecules (Temeeyasen et al. 2018). Whether the continuous deletion of 194 amino acids occurred

342 in Japanese strains will affect the virulence of these strains are unknown and warrant further

343 exploration. A previous study however has found that a Japanese strain Tottori2, which had the

344 same deletion, had non-lethal effects in piglets (Masuda et al. 2015). The mutations also were

345 found in C domain of S protein, since the NTD and C-domain both can bind to the host cell receptor 
346 and function as the receptor-binding domain, the amino acid changes in their sequences may have

347 important role for the virus ( $\mathrm{Li} 2012$ ). It has been reported the N-linked glycosylation sites on the

348 S protein of some coronaviruses such as SARS-CoV play a critical role in the viral entry (Han et

349 al. 2007). The phylogenetic and N-linked glycosylation sites analysis of S protein may offer

350 reasons for further studies. There was no too many mutations were found in the ORF3-E-M-N

351 proteins, it might be because some of them, such as E protein, do not bear too much immune

352 selective pressure since it has no effect on the host cell growth or cell cycle (Xu et al. 2013).

\section{CONCLUSIONS}

354 In conclusion, through virus isolation and complete genome sequencing, we obtained PEDV

355 HB2018 strain. Using this virus, we investigated the genetic and phylogenetic characteristics of

356 PEDV isolates in China as well as in Asia in this study. Phylogenetic analysis revealed

357 heterogeneous genotypes of PEDVs circulate in Asia, but GII particularly GII-a genotype 358 represents the main epidemic genotype in the continent. Our study also revealed that most of the 359 PEDVs currently prevalent in Asian countries displayed a different genotype as well as a distant 360 relationship from the conventional vaccine strain CV777. This finding might explain why CV777-

361 derived vaccine provided poor protection against PEDV epidemics (variant strains) since 2010. In

362 addition, we also identified many mutations in the S, ORF3, E, M, N proteins of the variant strains

363 (GII) compared to those of the classical strains (Temeeyasen et al.). The presence of these 364 mutations, particularly those determined in the $\mathrm{S}$ proteins, may affect the antigenicity, 365 pathogenicity, and neutralization properties of the variant strains.

\section{ADDITIONAL INFORMATION AND DECLARATIONS}




\section{Funding}

368 This work was supported by the Key Laboratory of Prevention and Control Agents for Animal

369 Bacteriosis (Ministry of Agriculture) (grant number: KLPCAAB-YTP-1801), the open funds of

370 the Key Laboratory of Preventive Veterinary Medicine of Hubei Province, and China Postdoctoral

371 Science foundation (grant number: 2019M652609). There was no additional external funding

372 received for this study.

373 Competing Interests

374 The authors declare there are no competing interests.

375 Acknowledgments

376 The authors sincerely appreciate Dr. Zhong Peng at College of Veterinary Medicine,

377 Huazhong Agricultural University, Wuhan, China for the language reversion and suggestions on

378 the analysis.

379

380

381

382

383

384

385

386

387 the accession number of MT166307. 
388 Supplemental Materials

389 Figure S1: RT-PCR detection of the viral nucleic acids from the intestinal samples of the pigs

390 suffered and/or died from severe watery diarrhea.

391 Figure S2: Sequence alignments on the whole genomes of HB2018 and CV777.

392 Figure S3: Sequence alignments on the S proteins of HB2018 and CV777.

393 Table S1: List of the 207 Asian PEDV isolates.

394 Txt S1: Alignment of the S protein of GI-a and GI-b strains from China.

395 Txt S2: Alignment of the S protein of Asian GII-a strains.

396 Txt S3: Alignment of the S protein of Asian GII-b strains.

\section{REFERENCES}

398

399

400

401

402

403

404

405

406

407

408

409

410

411

412

413

Aziz RK, Bartels D, Best AA, DeJongh M, Disz T, Edwards RA, Formsma K, Gerdes S, Glass EM, Kubal M, Meyer F, Olsen GJ, Olson R, Osterman AL, Overbeek RA, McNeil LK, Paarmann D, Paczian T, Parrello B, Pusch GD, Reich C, Stevens R, Vassieva O, Vonstein V, Wilke A, and Zagnitko O. 2008. The RAST Server: rapid annotations using subsystems technology. BMC Genomics 9:75. 10.1186/1471-2164-9-75

Bankevich A, Nurk S, Antipov D, Gurevich AA, Dvorkin M, Kulikov AS, Lesin VM, Nikolenko SI, Pham S, Prjibelski AD, Pyshkin AV, Sirotkin AV, Vyahhi N, Tesler G, Alekseyev MA, and Pevzner PA. 2012. SPAdes: a new genome assembly algorithm and its applications to single-cell sequencing. J Comput Biol 19:455-477. $10.1089 / \mathrm{cmb} .2012 .0021$

Bouckaert R, Vaughan TG, Barido-Sottani J, Duchêne S, Fourment M, Gavryushkina A, Heled J, Jones G, Kühnert D, De Maio N, Matschiner M, Mendes FK, Müller NF, Ogilvie HA, du Plessis L, Popinga A, Rambaut A, Rasmussen D, Siveroni I, Suchard MA, Wu CH, Xie D, Zhang C, Stadler T, and Drummond AJ. 2019. BEAST 2.5: An advanced software platform for Bayesian evolutionary analysis. PLoS Comput Biol 15:e1006650. 10.1371/journal.pcbi.1006650

Chen P, Wang K, Hou Y, Li H, Li X, Yu L, Jiang Y, Gao F, Tong W, Yu H, Yang Z, Tong G, and Zhou Y. 2019a. Genetic evolution analysis and pathogenicity assessment of porcine epidemic diarrhea virus strains circulating in part of China during 2011-2017. Infect Genet Evol 69:153-165. 10.1016/j.meegid.2019.01.022 
414 Chen X, Zhang XX, Li C, Wang H, Wang H, Meng XZ, Ma J, Ni HB, Zhang X, Qi Y, and Sun D. 2019b. Epidemiology 415 of porcine epidemic diarrhea virus among Chinese pig populations: A meta-analysis. Microb Pathog 129:43-49. $416 \quad$ 10.1016/j.micpath.2019.01.017

417 Choudhury B, Dastjerdi A, Doyle N, Frossard JP, and Steinbach F. 2016. From the field to the lab - An European view 418 on the global spread of PEDV. Virus Res 226:40-49. 10.1016/j.virusres.2016.09.003

419 Darling AC, Mau B, Blattner FR, and Perna NT. 2004. Mauve: multiple alignment of conserved genomic sequence with 420 rearrangements. Genome Res 14:1394-1403. 10.1101/gr.2289704

421

422

423

424

425

426

427

428

429

430

431

432

433

434

435

436

437

438

439

440

441

442

443

444

445

446

447

448

449

450

451
Gatto L, Catanzaro D, and Milinkovitch MC. 2007. Assessing the applicability of the GTR nucleotide substitution model through simulations. Evol Bioinform Online 2:145-155.

Gong L, Lin Y, Qin J, Li Q, Xue C, and Cao Y. 2018. Neutralizing antibodies against porcine epidemic diarrhea virus block virus attachment and internalization. Virol J 15:133. 10.1186/s12985-018-1042-3

Goris J, Konstantinidis KT, Klappenbach JA, Coenye T, Vandamme P, and Tiedje JM. 2007. DNA-DNA hybridization values and their relationship to whole-genome sequence similarities. Int J Syst Evol Microbiol 57:81-91. 10.1099/ijs.0.64483-0

Guo J, Fang L, Ye X, Chen J, Xu S, Zhu X, Miao Y, Wang D, and Xiao S. 2019. Evolutionary and genotypic analyses of global porcine epidemic diarrhea virus strains. Transbound Emerg Dis 66:111-118. 10.1111/tbed.12991

Han DP, Lohani M, and Cho MW. 2007. Specific asparagine-linked glycosylation sites are critical for DC-SIGN- and LSIGN-mediated severe acute respiratory syndrome coronavirus entry. J Virol 81:12029-12039. 10.1128/jvi.00315-07

Hou Y, Lin CM, Yokoyama M, Yount BL, Marthaler D, Douglas AL, Ghimire S, Qin Y, Baric RS, Saif LJ, and Wang Q. 2017. Deletion of a 197-Amino-Acid Region in the N-Terminal Domain of Spike Protein Attenuates Porcine Epidemic Diarrhea Virus in Piglets. J Virol 91. 10.1128/jvi.00227-17

Hsu TH, Liu HP, Chin CY, Wang C, Zhu WZ, Wu BL, and Chang YC. 2018. Detection, sequence analysis, and antibody prevalence of porcine deltacoronavirus in Taiwan. Arch Virol 163:3113-3117. 10.1007/s00705-018-3964-x

Hsueh FC, Lin CN, Chiou HY, Chia MY, Chiou MT, Haga T, Kao CF, Chang YC, Chang CY, Jeng CR, and Chang HW. 2020. Updated phylogenetic analysis of the spike gene and identification of a novel recombinant porcine epidemic diarrhoea virus strain in Taiwan. Transbound Emerg Dis 67:417-430. 10.1111/tbed.13365

Katoh K, and Standley DM. 2013. MAFFT multiple sequence alignment software version 7: improvements in performance and usability. Mol Biol Evol 30:772-780. 10.1093/molbev/mst010

Kumar S, Stecher G, Li M, Knyaz C, and Tamura K. 2018. MEGA X: Molecular Evolutionary Genetics Analysis across Computing Platforms. Mol Biol Evol 35:1547-1549. 10.1093/molbev/msy096

Letunic I, and Bork P. 2019. Interactive Tree Of Life (iTOL) v4: recent updates and new developments. Nucleic Acids Res 47:W256-w259. 10.1093/nar/gkz239

Li D, Feng H, Liu Y, Chen Y, Wei Q, Wang J, Liu D, Huang H, Su Y, Wang D, Cui Y, and Zhang G. 2018. Molecular evolution of porcine epidemic diarrhea virus and porcine deltacoronavirus strains in Central China. Res Vet Sci 120:63-69. 10.1016/j.rvsc.2018.06.001

Li F. 2012. Evidence for a common evolutionary origin of coronavirus spike protein receptor-binding subunits. J Virol 86:2856-2858. 10.1128/jvi.06882-11

Peer) reviewing PDF | (2020:03:47007:3:1:NEW 10 Sep 2020) 
452 Li W, Li H, Liu Y, Pan Y, Deng F, Song Y, Tang X, and He Q. 2012. New variants of porcine epidemic diarrhea virus,

453

454

455

456

457

458

459

460

461

462

463

464

465

466

467

468

469

470

471

472

473

474

475

476

477

478

479

480

481

482

483

484

485

486

487

488

489 China, 2011. Emerg Infect Dis 18:1350-1353. 10.3201/eid1808.120002

Lole KS, Bollinger RC, Paranjape RS, Gadkari D, Kulkarni SS, Novak NG, Ingersoll R, Sheppard HW, and Ray SC. 1999. Full-length human immunodeficiency virus type 1 genomes from subtype C-infected seroconverters in India, with evidence of intersubtype recombination. Journal of virology 73:152-160.

Masuda T, Murakami S, Takahashi O, Miyazaki A, Ohashi S, Yamasato H, and Suzuki T. 2015. New porcine epidemic diarrhoea virus variant with a large deletion in the spike gene identified in domestic pigs. Arch Virol 160:25652568. 10.1007/s00705-015-2522-z

Peng Z, Liang W, Liu W, Wu B, Tang B, Tan C, Zhou R, and Chen H. 2016. Genomic characterization of Pasteurella multocida HB01, a serotype A bovine isolate from China. Gene 581:85-93. 10.1016/j.gene.2016.01.041

Puranaveja S, Poolperm P, Lertwatcharasarakul P, Kesdaengsakonwut S, Boonsoongnern A, Urairong K, Kitikoon P, Choojai P, Kedkovid R, Teankum K, and Thanawongnuwech R. 2009. Chinese-like strain of porcine epidemic diarrhea virus, Thailand. Emerg Infect Dis 15:1112-1115. 10.3201/eid1507.081256

Reed LJ, and Muench H. 1983. A simple method of estimating fifty per cent endpoints 12. Am J Epidemiol 27:493-497.

Rocha EP, Smith JM, Hurst LD, Holden MT, Cooper JE, Smith NH, and Feil EJ. 2006. Comparisons of dN/dS are time dependent for closely related bacterial genomes. J Theor Biol 239:226-235. 10.1016/j.jtbi.2005.08.037

Sagesser R, Martinez E, Tsagris M, and Tabler M. 1997. Detection and isolation of RNA-binding proteins by RNAligand screening of a cDNA expression library. Nucleic Acids Res 25:3816-3822. 10.1093/nar/25.19.3816

Su Y, Hou Y, Prarat M, Zhang Y, and Wang Q. 2018. New variants of porcine epidemic diarrhea virus with large deletions in the spike protein, identified in the United States, 2016-2017. Arch Virol 163:2485-2489. 10.1007/s00705-018-3874-y

Su Y, Hou Y, and Wang Q. 2019. The enhanced replication of an S-intact PEDV during coinfection with an S1 NTD-del PEDV in piglets. Vet Microbiol 228:202-212. 10.1016/j.vetmic.2018.11.025

Sun J, Li Q, Shao C, Ma Y, He H, Jiang S, Zhou Y, Wu Y, Ba S, Shi L, Fang W, Wang X, and Song H. 2018. Isolation and characterization of Chinese porcine epidemic diarrhea virus with novel mutations and deletions in the $S$ gene. Vet Microbiol 221:81-89. 10.1016/j.vetmic.2018.05.021

Sun RQ, Cai RJ, Chen YQ, Liang PS, Chen DK, and Song CX. 2012. Outbreak of porcine epidemic diarrhea in suckling piglets, China. Emerg Infect Dis 18:161-163. 10.3201/eid1801.111259

Sun Y, Chen Y, Han X, Yu Z, Wei Y, and Zhang G. 2019. Porcine epidemic diarrhea virus in Asia: An alarming threat to the global pig industry. Infect Genet Evol 70:24-26. 10.1016/j.meegid.2019.02.013

Suzuki T, Terada Y, Enjuanes L, Ohashi S, and Kamitani W. 2018. S1 Subunit of Spike Protein from a Current Highly Virulent Porcine Epidemic Diarrhea Virus Is an Important Determinant of Virulence in Piglets. Viruses 10. 10.3390/v10090467

Tamura K, and Nei M. 1993. Estimation of the number of nucleotide substitutions in the control region of mitochondrial DNA in humans and chimpanzees. Mol Biol Evol 10:512-526. 10.1093/oxfordjournals.molbev.a040023

Temeeyasen G, Sinha A, Gimenez-Lirola LG, Zhang JQ, and Pineyro PE. 2018. Differential gene modulation of patternrecognition receptor TLR and RIG-I-like and downstream mediators on intestinal mucosa of pigs infected with PEDV non S-INDEL and PEDV S-INDEL strains. Virology 517:188-198. 10.1016/j.virol.2017.11.024

Peer) reviewing PDF | (2020:03:47007:3:1:NEW 10 Sep 2020) 
490

491

492

493

494

495

496

497

498

499

500

501

502

503

504

505

506

507

508

509

510

511

512

513

514

515

516

517
Vlasova AN, Marthaler D, Wang Q, Culhane MR, Rossow KD, Rovira A, Collins J, and Saif LJ. 2014. Distinct characteristics and complex evolution of PEDV strains, North America, May 2013-February 2014. Emerg Infect Dis 20:1620-1628. 10.3201/eid2010.140491

Wang D, Fang L, and Xiao S. 2016a. Porcine epidemic diarrhea in China. Virus Res 226:7-13. 10.1016/j.virusres.2016.05.026

Wang E, Guo D, Li C, Wei S, Wang Z, Liu Q, Zhang B, Kong F, Feng L, and Sun D. 2016b. Molecular Characterization of the ORF3 and S1 Genes of Porcine Epidemic Diarrhea Virus Non S-INDEL Strains in Seven Regions of China, 2015. PLoS One 11:e0160561. 10.1371/journal.pone.0160561

Wang L, Byrum B, and Zhang Y. 2014. Detection and genetic characterization of deltacoronavirus in pigs, Ohio, USA, 2014. Emerg Infect Dis 20:1227-1230. 10.3201/eid2007.140296

Woo PC, Lau SK, Lam CS, Lau CC, Tsang AK, Lau JH, Bai R, Teng JL, Tsang CC, Wang M, Zheng BJ, Chan KH, and Yuen KY. 2012. Discovery of seven novel Mammalian and avian coronaviruses in the genus deltacoronavirus supports bat coronaviruses as the gene source of alphacoronavirus and betacoronavirus and avian coronaviruses as the gene source of gammacoronavirus and deltacoronavirus. J Virol 86:3995-4008. 10.1128/jvi.06540-11

Xu X, Zhang H, Zhang Q, Dong J, Liang Y, Huang Y, Liu HJ, and Tong D. 2013. Porcine epidemic diarrhea virus E protein causes endoplasmic reticulum stress and up-regulates interleukin-8 expression. Virol J 10:26. $10.1186 / 1743-422 x-10-26$

Yang X, Huo JY, Chen L, Zheng FM, Chang HT, Zhao J, Wang XW, and Wang CQ. 2013. Genetic variation analysis of reemerging porcine epidemic diarrhea virus prevailing in central China from 2010 to 2011. Virus Genes 46:337344. 10.1007/s11262-012-0867-x

Zhang L, Liu X, Zhang Q, Zhou P, Fang Y, Dong Z, Zhao D, Li W, Feng J, Zhang Y, and Wang Y. 2019. Biological characterization and pathogenicity of a newly isolated Chinese highly virulent genotype GIIa porcine epidemic diarrhea virus strain. Arch Virol 164:1287-1295. 10.1007/s00705-019-04167-3

Zhang X, Pan Y, Wang D, Tian X, Song Y, and Cao Y. 2015. Identification and pathogenicity of a variant porcine epidemic diarrhea virus field strain with reduced virulence. Virol J 12:88. 10.1186/s12985-015-0314-4

Zhou YJ, Wu YL, Zhu JP, Tong W, Yu H, Jiang YF, and Tong GZ. 2012. Complete genome sequence of a virulent porcine epidemic diarrhea virus strain. J Virol 86:13862. 10.1128/jvi.02635-12

518 
Figure 1

Phylogenetic and genetic characteristics of PEDV strain HB2018.

(A) Phylogenetic analysis of HB2018 and the other PEDV strains based on the whole genome sequence; (B) Nucleotide similarity of the complete genome sequences between PEDV strains HB2018 and CV777; (C) Sequence alignment of the S-NTD regions of PEDV strains HB2018 and CV777; (D) Modelling the 3D structure of the S protein of CV777; (E) Modelling the 3D structure of the S protein of HB2018. 
A.

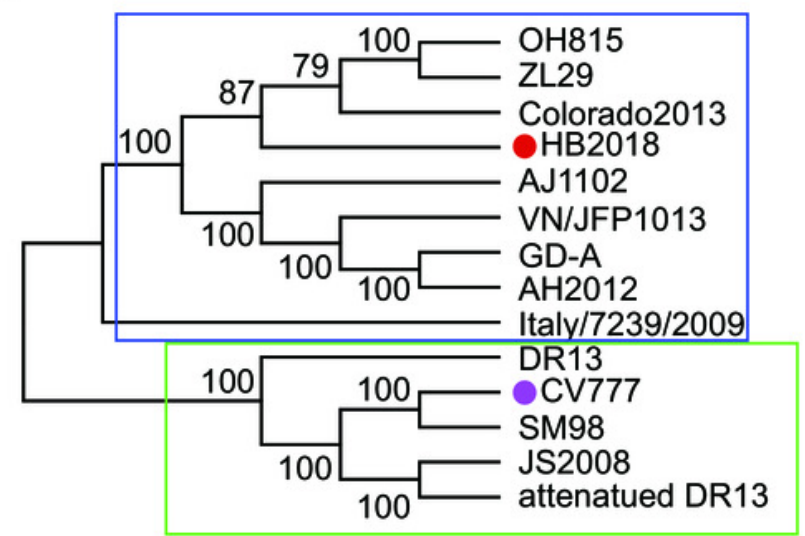

B.

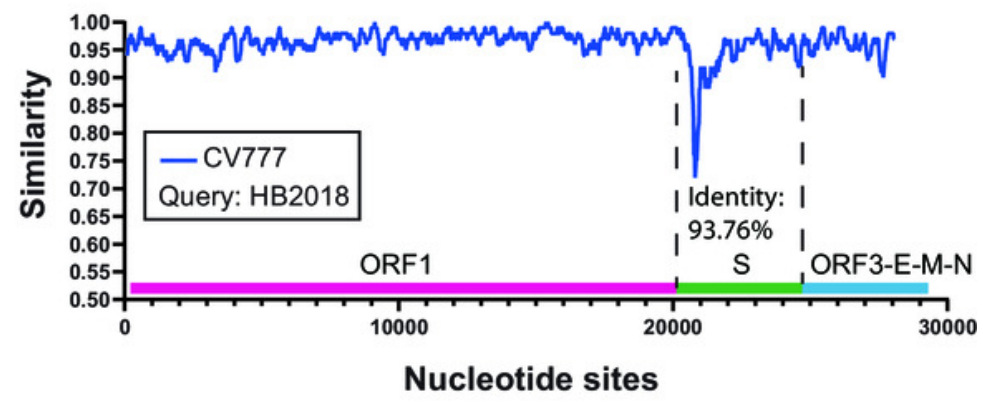

C.

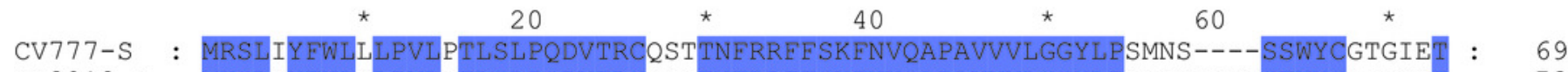
HB2018-S : MKSLTYFWLFLPVLSTLSLPQDVTRCSANTNERREFSKENVQAPAVVVLGGYLPIGENQGVNSTWYCAGQHPI : 73

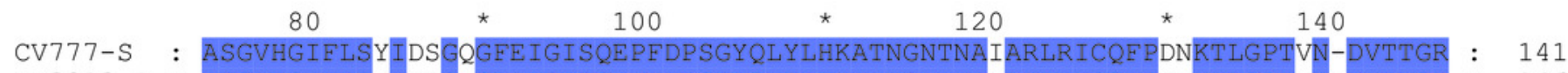
HB2018-S : ASGVHGIFLSHIRGGHGEEIGISQEPFDPSGYQLYLHKATNGNTNATARLRICQFPGIKTLGPTADNDVTTGR : 146

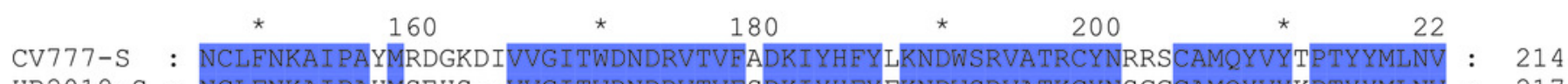
HB2018-S : NCLENKAIPAHMSEHS--VVGITWDNDRVTVESDKIYHFYFKNDWSRVATKCYNSGGCAMQYVYKPTYYMLNV : 217

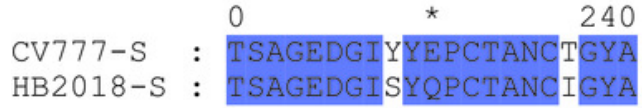

D.

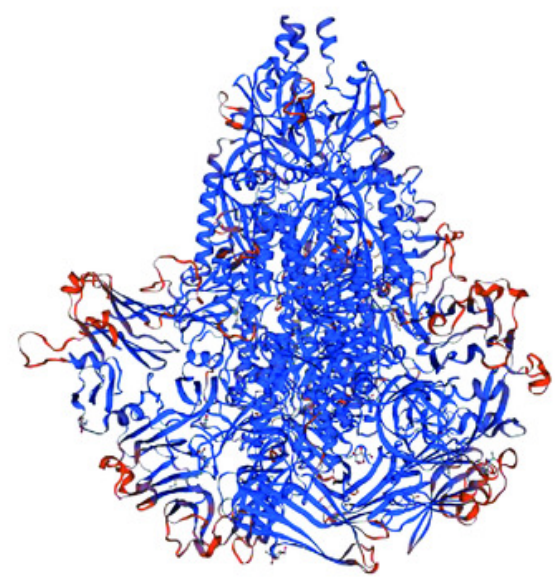

CV777
E.

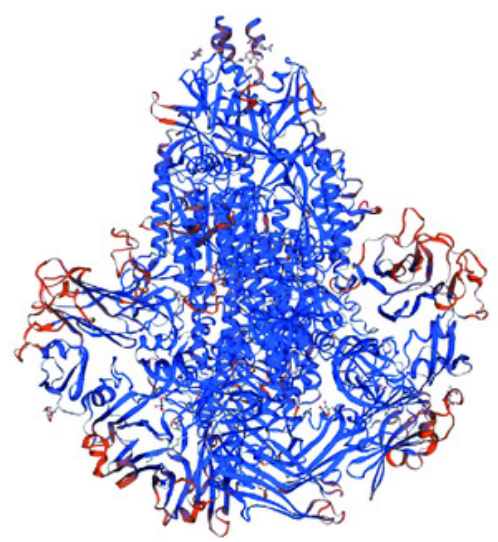

HB2018 


\section{Figure 2}

Genotyping of the 208 Asian PEDV strains and the non-Asian reference strains based on full-length genomic sequences.

(A) A phylogenetic tree was generated by using the MEGAX package. The evolutionary history was inferred by using the Maximum Likelihood method based on the TN93 model. The tree is drawn to scale, with branch lengths measured in the number of substitutions per site. GenBank accession numbers of strains, years, places of isolation, genogroups, and subgroups are shown. (B) A Maximum Likelihood tree was generated by using the BEAST 2 package. Gamma correction for site heterogeneity and the GTR model were selected for the tree generation. GenBank accession numbers of strains, years, places of isolation, genogroups, and subgroups are shown. (C) Line chart shows the number of PEDV sequences obtained by gene subgroup and year of sampling. Yearly percentages of samples positive for PEDV are indicated by different colored lines. 

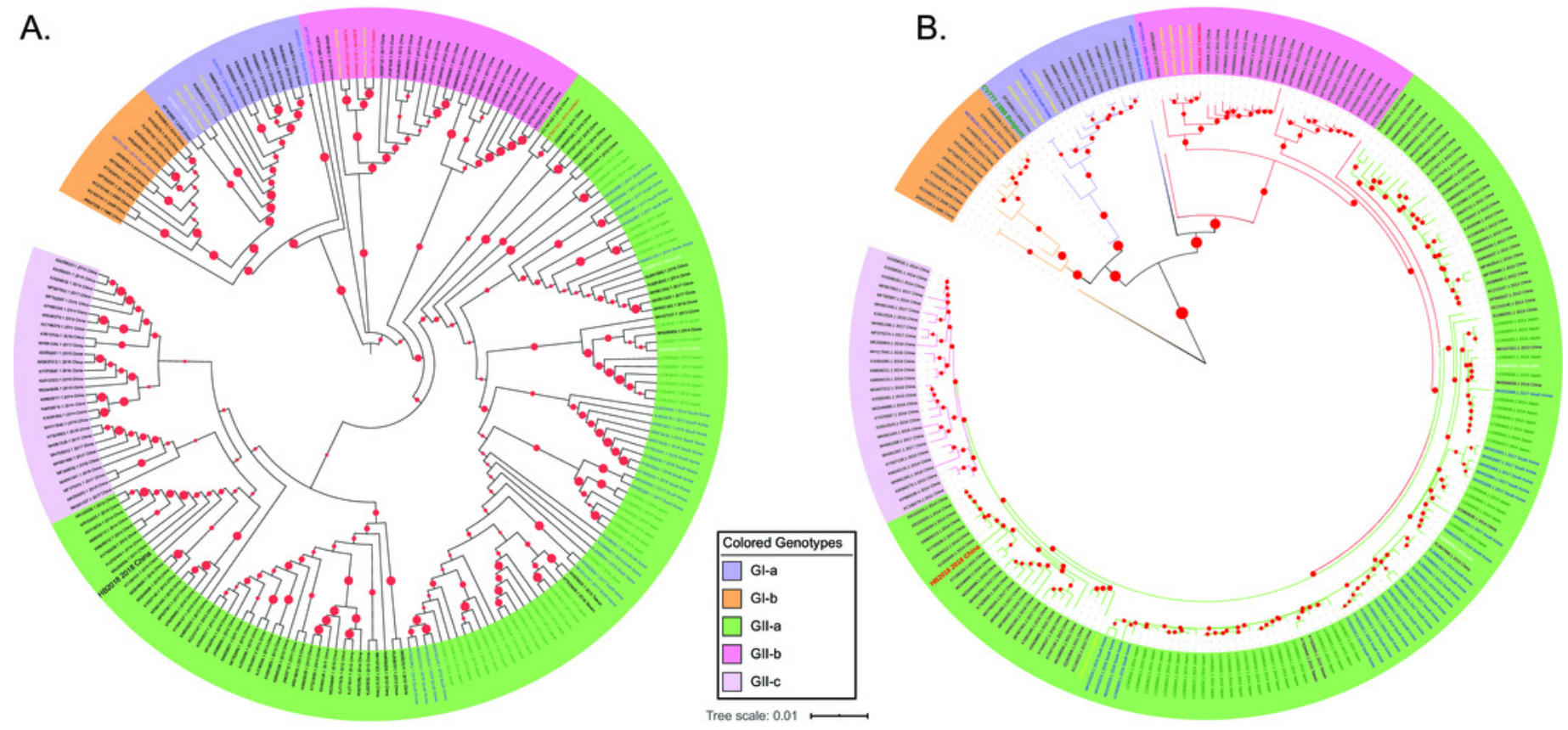

C.

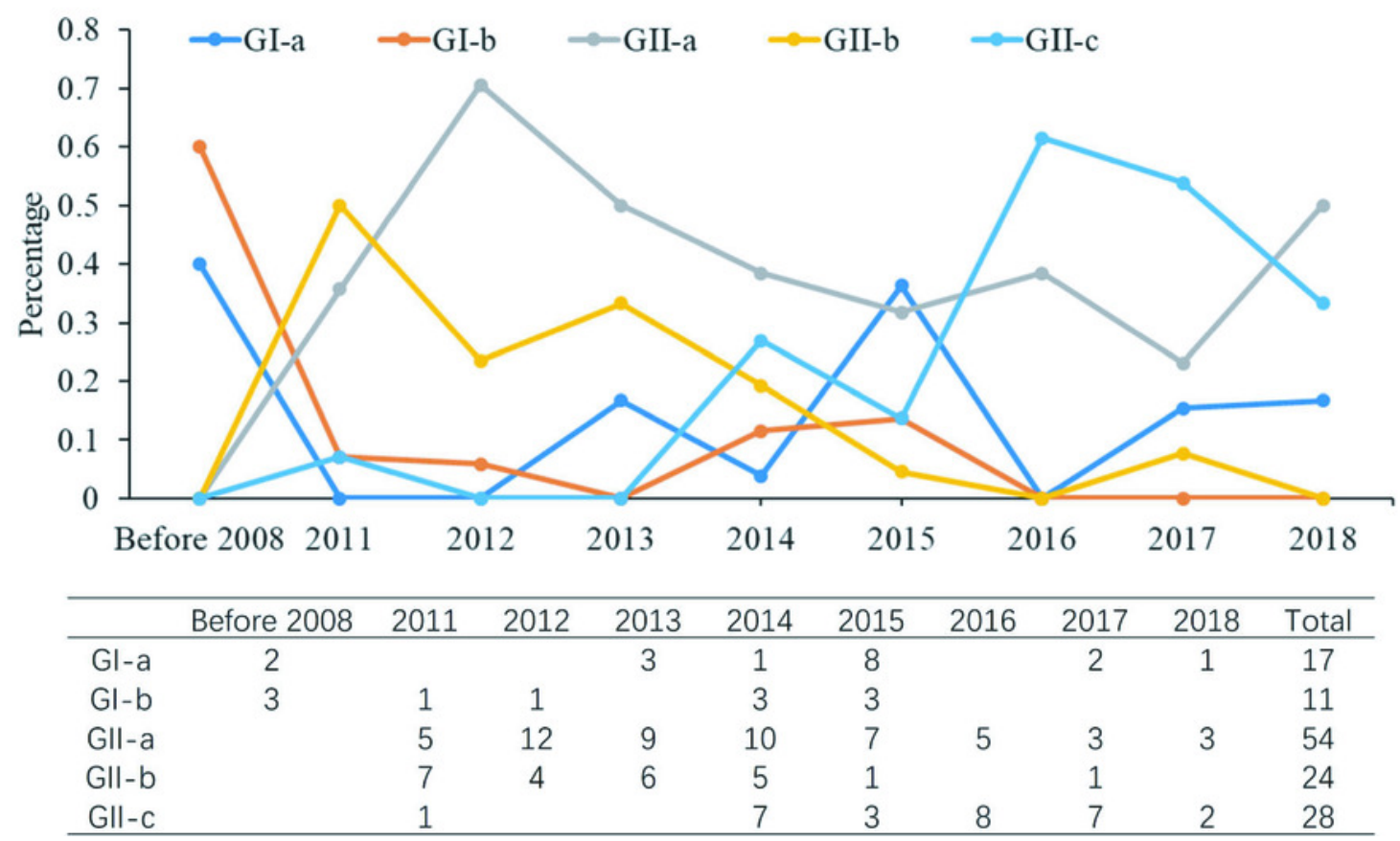


Figure 3

Sequence alignment of the S-NTD regions of PEDV strains.

(A) Sequence alignment of the S-NTD regions of the strains from China; (B) Sequence alignment of the S-NTD regions of the strains from Asia.

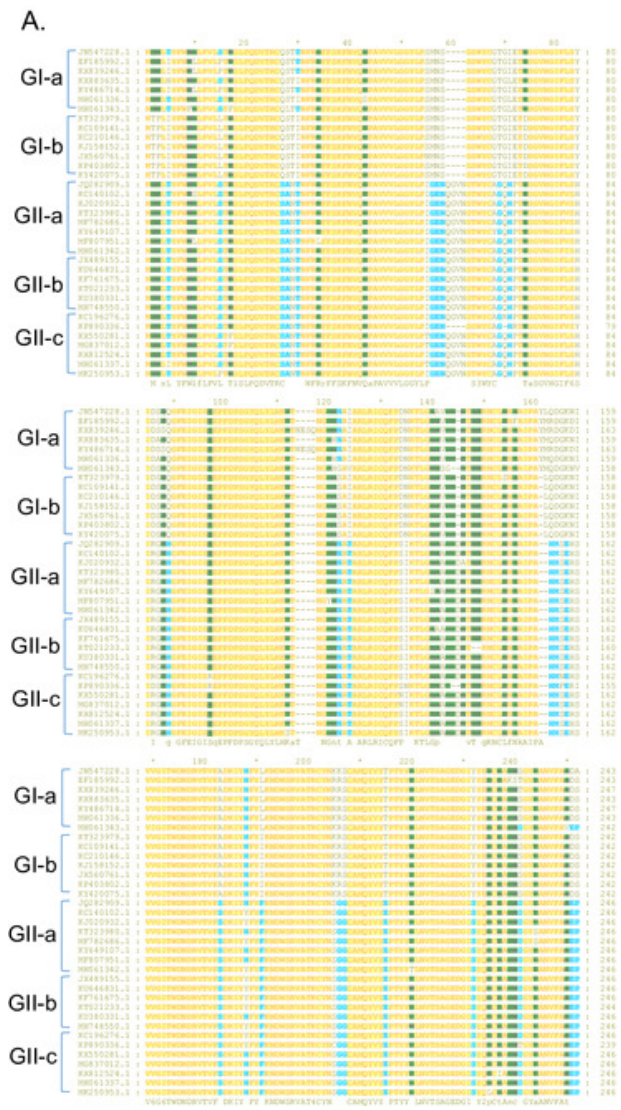

B.
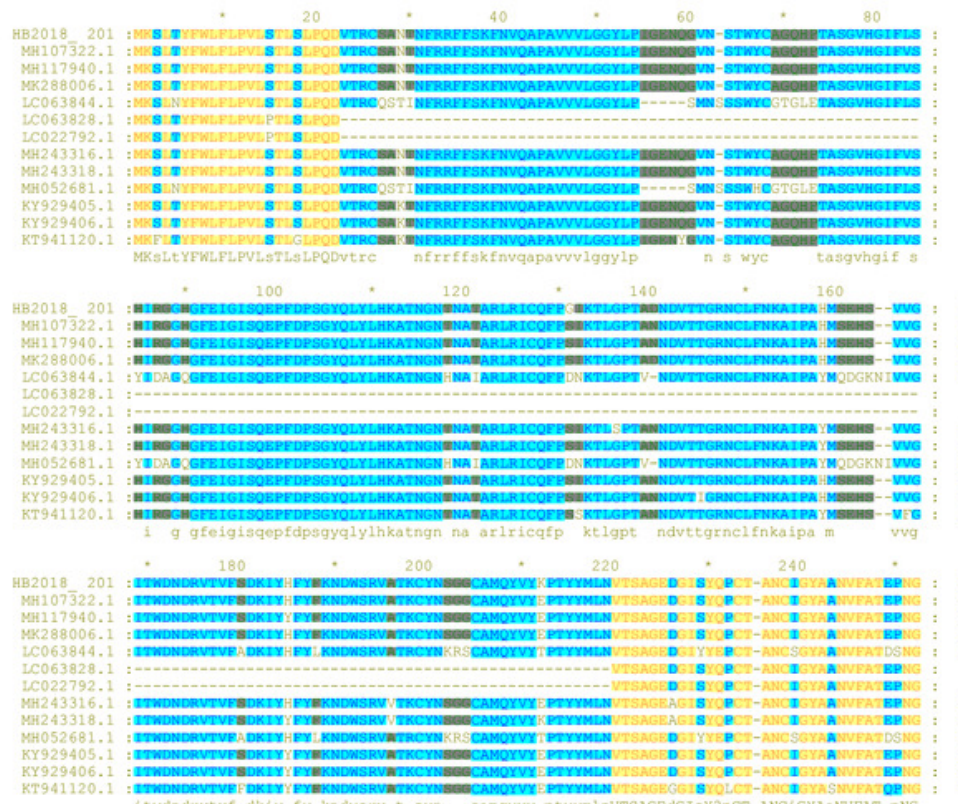
Figure 4

\section{Sequence alignment of ORF3 of PEDV strains.}

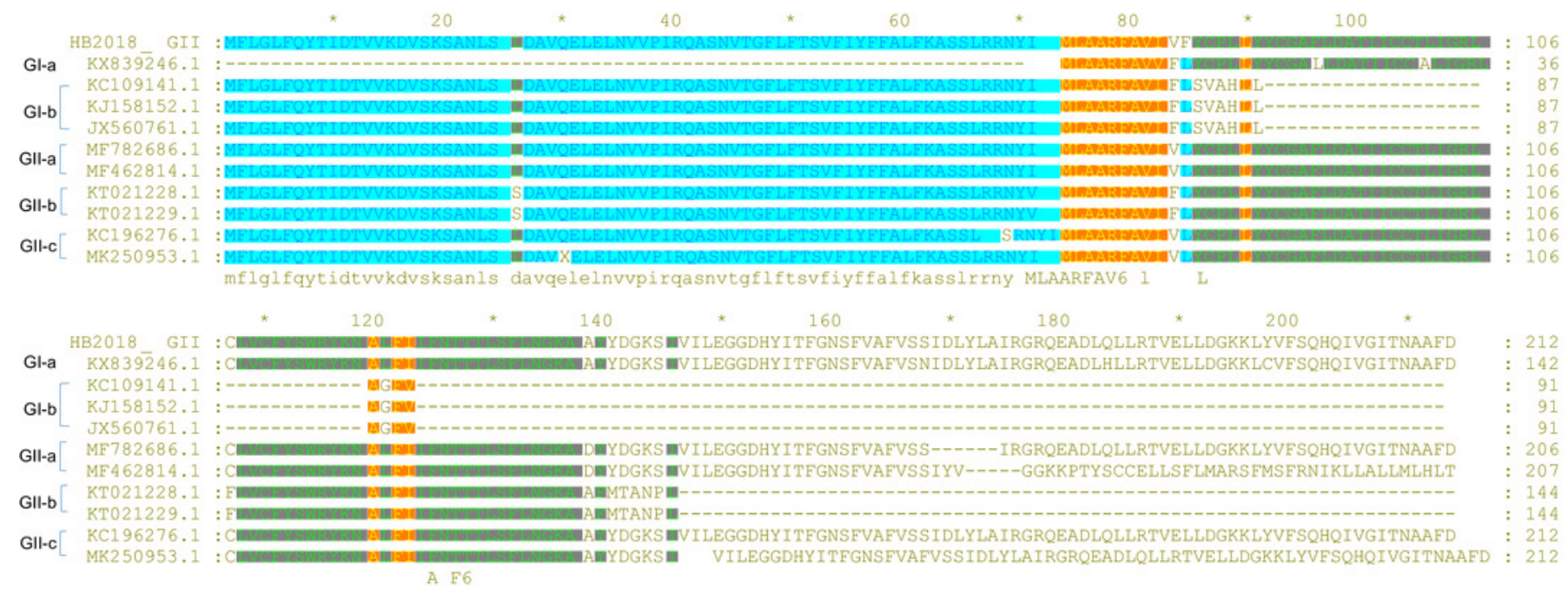


Figure 5

\section{Sequence alignment of $E, M$ and $N$ protein of PEDV strains.}

(A) E protein; (B) M protein; (C) N protein.

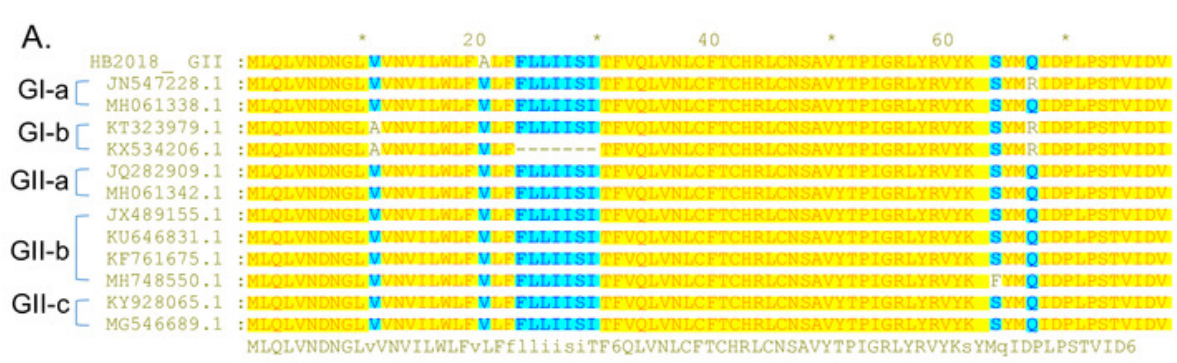

B.

20

40

60

80

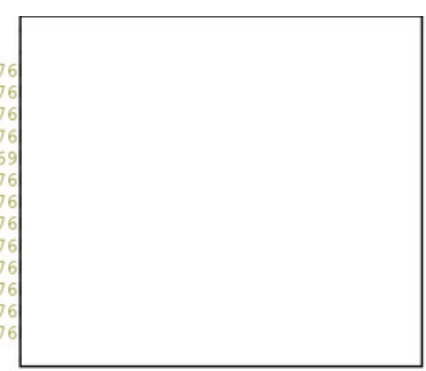

MSNGS PVDEVIOHLRNWNFTWNITLTILLVVLOYGHYKYSVFLYGVKMATLWTIW WLWLALSLFDAWASFOVNWVFFAFCLU

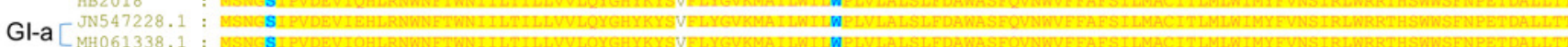

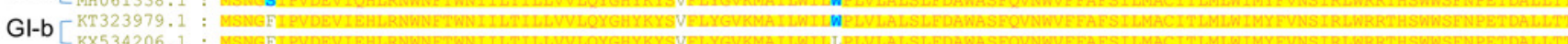

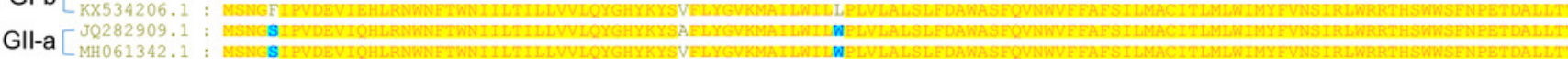

$-\mathrm{JX} 489155.1$

GII-b KU646831

GII-C

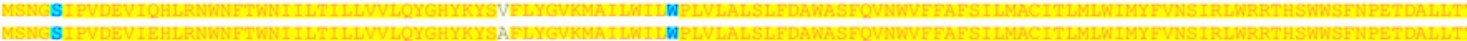

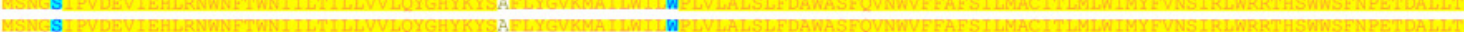

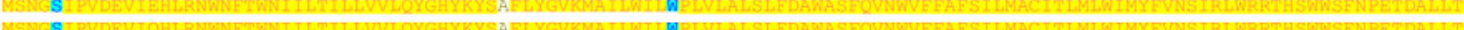

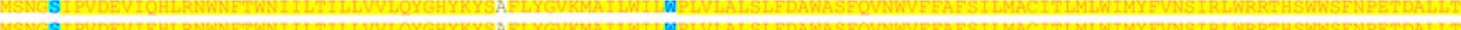

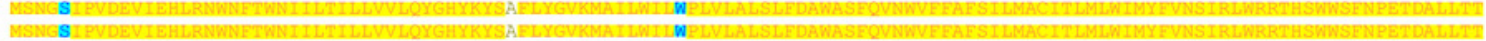

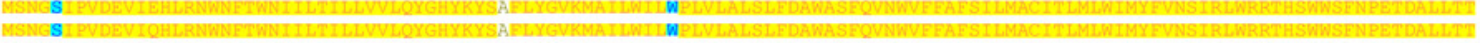
MSNGS I PVDEVI HLRNWNETWNI I LTI LLVVLQYGHYKYS ELYGVKMA I LWI LW PLVLALSLF DAWASFQVNWVFFAFSI LMACITLMLWIMYFVNSIRLWRRTHSWWSFNPET DALLTT

HB2018

$\mathrm{Gl}-\mathrm{a}-\mathrm{JN} 54722$

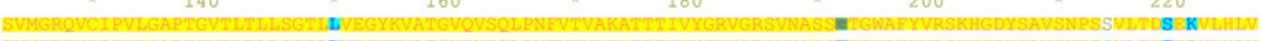

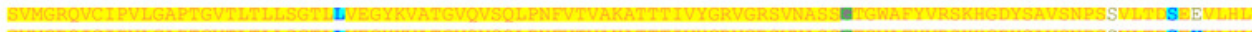

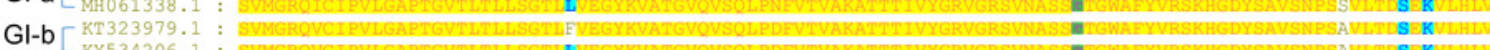

Gl-b [ KT K 323979.1

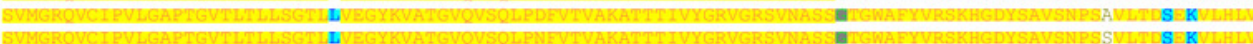

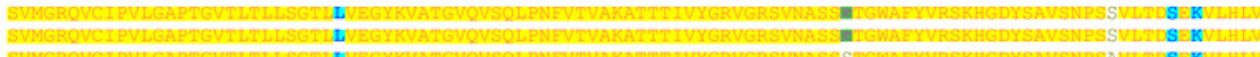

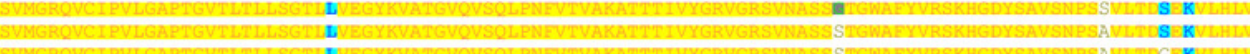
SUMGROVCIPVLGAPTGVTLTLLSGTLAVEGYKVATGVQVSOLPNEVTVAKATTTIVYGRVGRSVNASSSTGWAFYVRSKHGDYSAVSWPSAVLIDGEKVLHLV

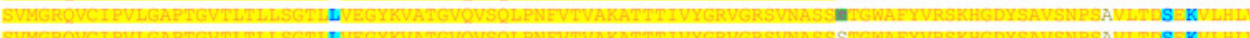

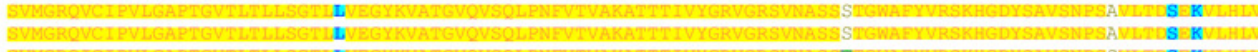

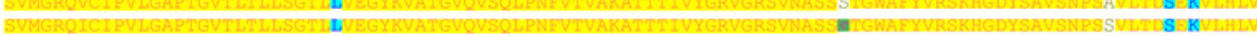
SVMGRQ6CIPVLGAPTGVTLTLLSGTLIVEGYKVATGVQVSQLP1FVTVAKATTTIVYGRVGRSVNASS TGWAFYVRSKHGDYSAVSNPS VLTDSEKVLHLV

C.

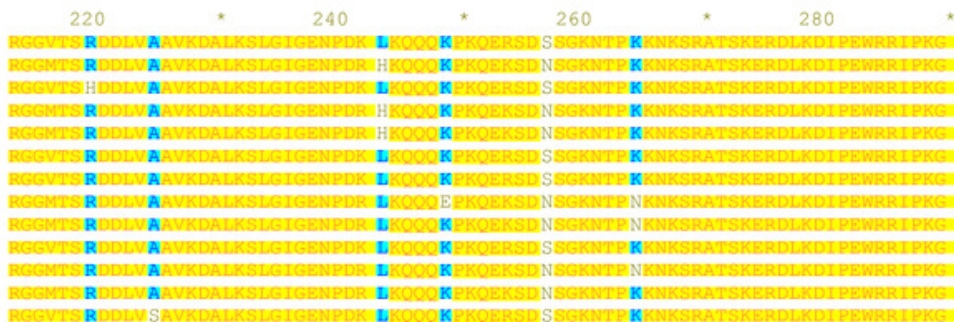

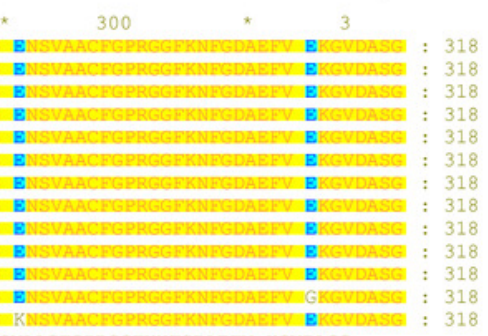
RGG6TSRDDLVAAVKDALKSLGIGENPD41KQQQKPKQE4SD SGKNTPkKNKSRATSKERDLKDIPEWRRIPKGENSVAACFGPRGGFKNFGDAEFVEKGVDASG 


\section{Table 1 (on next page)}

Sequence comparisons of different ORF regions between HB2018 and CV777. 
Table 1. Sequence comparisons of different ORF regions between HB2018 and CV777.

\begin{tabular}{ccc}
\hline \multirow{2}{*}{ ORFs } & \multicolumn{2}{c}{ HB2018 vs. CV777 } \\
\cline { 2 - 3 } & Amino acid similarity (\%) & DNA identity (\%) \\
\hline ORF1 & 97.73 & 97.11 \\
\hline S & 93.44 & 93.76 \\
\hline ORF3 & 95.98 & 96.44 \\
\hline E & 97.40 & 96.97 \\
\hline M & 99.12 & 97.80 \\
\hline $\mathrm{N}$ & 96.60 & 95.48 \\
\hline
\end{tabular}

2 


\section{Table 2 (on next page)}

Single nucleotide polymorphism (SNP) analysis and dN/dS ratios of PEDV strains HB2018 and CV777. 
1 Table 2 Single nucleotide polymorphism (SNP) analysis and dN/dS ratios of PEDV strains

$2 \quad$ HB2018 and CV777.

\begin{tabular}{cccccc}
\hline & ORFs & Sum & $\begin{array}{c}\text { Non- } \\
\text { synonymous }\end{array}$ & Synonymous & dN/dS \\
\hline & Total & 925 & 262 & 663 & 0.395 \\
& ORF1a & 397 & 118 & 279 & 0.423 \\
& ORF1b & 188 & 26 & 162 & 0.160 \\
HB2018 vs. & S & 233 & 88 & 145 & 0.607 \\
CV777 & ORF3 & 24 & 9 & 15 & 0.600 \\
& E & 8 & 2 & 6 & 0.333 \\
& M & 15 & 2 & 13 & 0.154 \\
& N & 60 & 17 & 43 & 0.395 \\
\hline
\end{tabular}

3 


\section{Table 3 (on next page)}

High-specificity N-glycosylation sites predicted in Asian strains 
Table 3. High-specificity N-glycosylation sites predicted in Asian strains.

\begin{tabular}{|c|c|c|c|c|c|c|c|c|c|c|c|c|c|c|c|c|c|c|c|c|c|}
\hline \multirow{4}{*}{$\begin{array}{l}\text { Country/ } \\
\text { Regions }\end{array}$} & \multirow{4}{*}{ Strain } & \multicolumn{20}{|c|}{ High-specificity N-glycosylation sites ${ }^{1}$} \\
\hline & & 57 & 112 & 127 & 212 & 320 & 347 & 510 & 552 & 777 & 1245 & 1257 & & & & & & & & & \\
\hline & & NSTW & NAT & NKT & NVT & NDT & NSS & NIT & NVT & NIS & NKT & NRT & 22 & 347 & 382 & 421 & 524 & 739 & 869 & 1198 & 1274 \\
\hline & & NSTW & $\mathrm{A}$ & $\mathrm{L}$ & $\mathrm{s}$ & $\mathrm{s}$ & $\mathrm{D}$ & $\mathrm{V}$ & $\mathrm{N}$ & I & $\mathrm{L}$ & G & & & & & & & & & \\
\hline \multirow{10}{*}{ China } & CV777 & - & $\begin{array}{c}\text { NTS } \\
\text { A }\end{array}$ & • & • & - & $\cdot$ & • & • & $\cdot$ & $\cdot$ & - & - & - & - & - & - & - & - & - & - \\
\hline & HB2018 & • & $\cdot$ & - & • & • & • & - & - & $\cdot$ & $\cdot$ & - & - & - & - & - & - & - & - & - & - \\
\hline & LZC & - & - & $\cdot$ & $\cdot$ & $\cdot$ & • & - & $\cdot$ & $\cdot$ & $\cdot$ & $\cdot$ & - & - & - & - & - & - & - & - & $\begin{array}{c}\text { NLT } \\
\text { G }\end{array}$ \\
\hline & DR13 & • & • & - & • & • & $\begin{array}{c}\text { NSS } \\
\mathrm{N} \\
\end{array}$ & - & - & $\cdot$ & • & $\cdot$ & - & - & - & - & - & & - & - & - \\
\hline & SD-M & NSSS & $\begin{array}{c}\text { NTS } \\
\text { A }\end{array}$ & $\cdot$ & • & - & • & • & • & • & $\cdot$ & - & - & - & - & - & - & - & - & - & - \\
\hline & AJ1102 & • & $\cdot$ & - & • & $\cdot$ & $\cdot$ & $\cdot$ & - & $\cdot$ & $\cdot$ & $\cdot$ & - & - & - & - & - & - & - & - & - \\
\hline & FJZZ1 & • & $\cdot$ & - & • & • & $\cdot$ & $\cdot$ & - & $\cdot$ & $\cdot$ & $\cdot$ & - & - & - & - & - & - & - & - & - \\
\hline & $\mathbf{L S}$ & - & - & - & • & • & • & - & - & $\cdot$ & • & $\cdot$ & $\begin{array}{c}\text { NVT } \\
\text { R } \\
\end{array}$ & - & - & & - & - & - & - & - \\
\hline & CHS & - & & $\cdot$ & • & • & • & $\cdot$ & • & • & $\cdot$ & $\cdot$ & - & - & - & $\begin{array}{c}\text { NFT } \\
\text { D }\end{array}$ & - & - & - & - & - \\
\hline & $\begin{array}{c}\text { CHHNQX- } \\
314 \\
\end{array}$ & - & • & • & • & $\bullet$ & $\cdot$ & - & - & $\cdot$ & • & • & - & - & - & - & $\begin{array}{c}\text { NLT } \\
\mathrm{A} \\
\end{array}$ & $\begin{array}{c}\text { NCT } \\
\mathrm{E} \\
\end{array}$ & - & - & - \\
\hline \multirow{3}{*}{ Thailand } & CHYJ130330 & • & - & & • & • & $\begin{array}{c}\text { NST } \\
\mathrm{N} \\
\end{array}$ & $\cdot$ & - & $\begin{array}{c}\text { NIT } \\
\text { I } \\
\end{array}$ & • & • & - & - & - & - & - & - & - & - & - \\
\hline & CBR1 & • & $\cdot$ & $\cdot$ & • & - & $\cdot$ & $\cdot$ & - & $\cdot$ & $\cdot$ & $\cdot$ & - & - & - & - & - & - & - & - & - \\
\hline & AVCT12 & - & - & $\cdot$ & • & • & • & - & • & $\cdot$ & • & - & - & - & - & - & - & - & - & $\begin{array}{c}\text { NYT } \\
\text { A }\end{array}$ & - \\
\hline Taiwan & PT-P5 & • & • & - & • & • & $\begin{array}{c}\text { NSS } \\
\mathrm{N}\end{array}$ & • & - & - & $\begin{array}{c}\text { NKT } \\
\text { R }\end{array}$ & • & - & - & - & - & - & & - & - & - \\
\hline \multirow{3}{*}{$\begin{array}{l}\text { South } \\
\text { Korea }\end{array}$} & KNU-1709 & • & • & - & • & • & $\begin{array}{c}\text { NSS } \\
\mathrm{N} \\
\end{array}$ & - & - & $\cdot$ & • & • & - & - & $\begin{array}{c}\text { NST } \\
\mathrm{V} \\
\end{array}$ & - & - & - & $\begin{array}{c}\text { NIS } \\
\mathrm{S} \\
\end{array}$ & - & - \\
\hline & KNU-1702 & NSSS & - & - & $\cdot$ & $\cdot$ & $\cdot$ & - & - & $\cdot$ & • & • & - & - & - & - & - & - & - & - & - \\
\hline & KNU-1305 & $\cdot$ & $\cdot$ & - & • & • & $\begin{array}{c}\text { NSS } \\
\mathrm{N} \\
\end{array}$ & - & - & • & • & • & - & $\begin{array}{c}\mathrm{NFS} \\
\mathrm{L} \\
\end{array}$ & - & - & - & - & - & - & - \\
\hline Vietnam & HUA- & • & • & - & • & • & NSS & - & - & • & • & - & - & - & - & - & - & - & NIS & - & - \\
\hline
\end{tabular}




\section{PeerJ}

\begin{tabular}{|c|c|c|c|c|c|c|c|c|c|c|c|c|c|c|c|c|c|c|c|c|c|}
\hline & 14PED96 & & & & & & $\mathrm{N}$ & & & & & & & & & & & & $\mathrm{s}$ & & \\
\hline & VN/JFP1013 & - & - & • & • & - & $\cdot$ & - & - & • & - & - & - & - & - & - & - & - & - & - & - \\
\hline \multirow{3}{*}{ Japan } & Tottori2 & - & - & - & - & - & $\cdot$ & - & - & - & • & - & - & - & - & - & - & & - & $\begin{array}{c}\text { NYT } \\
\text { A }\end{array}$ & - \\
\hline & OKY-1 & - & - & $\cdot$ & $\cdot$ & - & - & • & $\cdot$ & - & • & - & - & - & - & - & - & - & - & - & - \\
\hline & IBR-7 & - & - & - & • & - & $\begin{array}{c}\text { NSS } \\
\text { N }\end{array}$ & - & - & - & • & - & - & - & - & - & - & - & - & - & - \\
\hline
\end{tabular}

21 The high-specificity N-glycosylation sites and their amino acids are summarized, the representative strains from each country/regions are listed." $\bullet$ means the strain has this high-specificity N-glycosylation site; "-" means the strain has no this high-specificity N-glycosylation site; the amino acid sequences means the strain has a high-specificity N-glycosylation in this sits, but the amino acid sequences are different with the common sequences. 